\title{
A SIMPLE DERIVATION OF JACOBI'S FOUR-SQUARE FORMULA
}

\author{
JOHN A. EWELL
}

\begin{abstract}
For each nonnegative integer $n, r_{4}(n)$ counts number of solutions $\left(x_{1}, x_{2}, x_{3}, x_{4}\right) \in \mathbf{Z}^{4}$ of $n=x_{1}^{2}+x_{2}^{2}+x_{3}^{2}+x_{4}^{2}$. Within the confines of elementary partition theory the author presents a simple derivation of Jacobi's formula for $r_{4}(n)$.
\end{abstract}

1. Introduction. In this investigation we take as our point of departure the following theorem.

THEOREM 1. For each nonnegative integer $n$, there exists a quadruple of integers $\left(x_{1}, x_{2}, x_{3}, x_{4}\right)$ such that

$$
n=x_{1}^{2}+x_{2}^{2}+x_{3}^{2}+x_{4}^{2} \text {. }
$$

This theorem is a celebrated result. As a matter of fact, in his classical History of the Theory of Numbers [1, pp. 275-303] L. E. Dickson considered the result so important that he devoted an entire chapter to its discussion. Following Dickson we briefly here record that the theorem was conjectured by Bachet in 1621, was claimed to have been proved by Fermat, but was not actually proved until Lagrange did so in 1770. It should also be mentioned that Lagrange was greatly assisted by Euler, who derived an identity which was crucial in Lagrange's argument. In this note we prove the stronger result of Jacobi, which for each given positive integer $n$ gives a count of the number of such representations of $n$. Since the count is expressed in terms of the sum-of-divisors function $\sigma$, we need the following definition.

DEFINITION. (i) For each positive integer $n, \sigma(n)$ denotes the sum of all positive divisors of $n$. (ii) For each nonnegative integer $n, r_{4}(n)$ denotes the cardinal number of the set

$$
\left\{\left(x_{1}, x_{2}, x_{3}, x_{4}\right) \in \mathbf{Z}^{4} \mid n=x_{1}^{2}+x_{2}^{2}+x_{3}^{2}+x_{4}^{2}\right\} .
$$

(iii) Each positive integer $n$ has a unique representation $n=2^{b(n)} O(n)$, where $b(n)$ is a nonnegative integer and $O(n)$ is odd.

We are now prepared to state our main result.

THEOREM 2. For each positive integer $n$,

$$
\begin{aligned}
r_{4}(2 n-1) & =8 \sigma(2 n-1), \\
r_{4}(2 n) & =24 \sigma(O(2 n)) .
\end{aligned}
$$

Received by the editors November 23, 1981.

1980 Mathematics Subject Classification. Primary 10J05; Secondary 10A45.

Key words and phrases. Four-square theorem, Jacobi's formula for $r_{4}(n)$.

(c) 1982 American Mathematical Society 
$\S 2$ is dedicated to the proof of Theorem 2. Our proof depends on the following three identities of partition theory.

$$
\begin{gathered}
\prod_{n=1}^{\infty}\left(1-x^{2 n}\right)\left(1+x^{2 n-1}\right)^{2}=\sum_{n=-\infty}^{\infty} x^{n^{2}} \\
2 \prod_{n=1}^{\infty} \frac{1-x^{2 n}}{1-x^{2 n-1}}=\sum_{n=-\infty}^{\infty} x^{n(n+1) / 2}, \\
\prod_{n=1}^{\infty}\left(1-x^{n}\right)^{6}=\sum_{-\infty}^{\infty} x^{n^{2}} \sum_{0}^{\infty}(2 n+1)^{2} x^{n(n+1)} \\
-\sum_{-\infty}^{\infty} x^{n(n+1)} \sum_{1}^{\infty}(2 n)^{2} x^{n^{2}} .
\end{gathered}
$$

These identities, and all that follow, are valid for each complex number $x$ such that $|x|<1$. (1) and (2) are due to Gauss. For proofs see [3, pp. 282-284]. For a proof of (3) see [2].

2. Proof of Theorem 2. First of all, we realize that the fourth power of the right side of identity (1) generates $r_{4}(n)$. Under the substitution $x \rightarrow-x$, identity (3) then becomes

$$
\begin{aligned}
\prod_{1}^{\infty} \frac{\left(1-x^{2 n}\right)^{2}}{\left(1+x^{2 n-1}\right)^{2}} \sum_{0}^{\infty} r_{4}(n) x^{n}= & \prod_{1}^{\infty} \frac{\left(1-x^{2 n}\right)^{2}}{\left(1+x^{2 n-1}\right)^{2}} \prod_{1}^{\infty}\left(1-x^{2 n}\right)^{4}\left(1+x^{2 n-1}\right)^{8} \\
= & \sum_{-\infty}^{\infty}(-1)^{n} x^{n^{2}} \sum_{0}^{\infty}(2 n+1)^{2} x^{n(n+1)} \\
& -\sum_{-\infty}^{\infty} x^{n(n+1)} \sum_{1}^{\infty}(-1)^{n}(2 n)^{2} x^{n^{2}}
\end{aligned}
$$

In the foregoing identity we let $x \rightarrow x^{4}$ and multiply the resulting identity by $x$ to get

$$
\begin{aligned}
x \prod_{1}^{\infty} \frac{\left(1-x^{8 n}\right)^{2}}{\left(1+x^{8 n-4}\right)^{2}} \sum_{0}^{\infty} r_{4}(n) x^{4 n}= & \sum_{-\infty}^{\infty}(-1)^{n} x^{4 n^{2}} \sum_{0}^{\infty}(2 n+1)^{2} x^{(2 n+1)^{2}} \\
& -\sum_{-\infty}^{\infty} x^{(2 n+1)^{2}} \sum_{1}^{\infty}(-1)^{n} 4 n^{2} x^{4 n^{2}}
\end{aligned}
$$

Now, let $F(x)$ be defined by

$$
\begin{aligned}
2 F(x) & =\frac{\sum_{-\infty}^{\infty} x^{(2 n+1)^{2}}}{\sum_{-\infty}^{\infty}(-1)^{n} x^{4 n^{2}}} \\
& =\frac{2 x \prod_{1}^{\infty} \frac{1-x^{16 n}}{1-x^{16 n-8}}}{\prod_{1}^{\infty}\left(1-x^{8 n}\right)\left(1-x^{8 n-4}\right)^{2}} \\
& =2 x \prod_{1}^{\infty}\left(1-x^{16 n-8}\right)^{-2}\left(1-x^{8 n-4}\right)^{-2} .
\end{aligned}
$$


Here, we have used identities (1) and (2) to obtain the infinite-product representation of $2 F(x)$. Hence, we differentiate the defining series representation of $F(x)$ with respect to $x$, and multiply the resulting identity by $x$ to get

$$
\begin{aligned}
x F^{\prime}(x)=\left\{\sum_{-\infty}^{\infty}(-1)^{n} x^{4 n^{2}}\right\}^{-2}\left\{\sum_{0}^{\infty}(2 n+1)^{2} x^{(2 n+1)^{2}} \sum_{-\infty}^{\infty}(-1)^{n} x^{4 n^{2}}\right. \\
\left.-\sum_{-\infty}^{\infty} x^{(2 n+1)^{2}} \sum_{1}^{\infty}(-1)^{n} 4 n^{2} x^{4 n^{2}}\right\} .
\end{aligned}
$$

Hence, the right side of (4) equals

$$
x\left\{\sum_{-\infty}^{\infty}(-1)^{n} x^{4 n^{2}}\right\}^{2} F^{\prime}(x)=x \prod_{1}^{\infty}\left(1-x^{8 n}\right)^{2}\left(1-x^{8 n-4}\right)^{4} F^{\prime}(x) .
$$

Substituting this expression into (4) and simplifying, we get

$$
\begin{aligned}
F^{\prime}(x) & =\prod_{1}^{\infty}\left(1+x^{8 n-4}\right)^{-2}\left(1-x^{8 n-4}\right)^{-4} \sum_{0}^{\infty} r_{4}(n) x^{4 n} \\
& =\prod_{1}^{\infty}\left(1-x^{16 n-8}\right)^{-2}\left(1-x^{8 n-4}\right)^{-2} \sum_{0}^{\infty} r_{4}(n) x^{4 n} \\
& =x^{-1} F(x) \sum_{0}^{\infty} r_{4}(n) x^{4 n} .
\end{aligned}
$$

We now use the technique to logarithmic differentiation on the infinite-product representation of $F(x)$ to express $F^{\prime}(x)$ in another way. To this end, we write

$$
\begin{aligned}
x D_{x}\{\log F(x)\} & =x \frac{F^{\prime}(x)}{F(x)} \\
& =1+16 \sum_{1}^{\infty} \frac{(2 n-1) x^{8(2 n-1)}}{1-x^{8(2 n-1)}}+8 \sum_{1}^{\infty} \frac{(2 n-1) x^{4(2 n-1)}}{1-x^{4(2 n-1)}} \\
& =1+16 \sum_{1}^{\infty} x^{8 n} \sum_{\substack{d \mid n \\
d \text { odd }}} d+8 \sum_{1}^{\infty} x^{4 n} \sum_{\substack{d \mid n \\
d \text { odd }}} d \\
& =1+16 \sum_{1}^{\infty} \sigma(O(n)) x^{8 n}+8 \sum_{1}^{\infty} \sigma(O(n)) x^{4 n} \\
& =1+\sum_{1}^{\infty}\{16 \sigma(O(n))+8 \sigma(O(2 n))\} x^{8 n}+8 \sum_{1}^{\infty} \sigma(2 n-1) x^{8 n-4} \\
& =1+24 \sum_{1}^{\infty} \sigma(O(2 n)) x^{8 n}+8 \sum_{1}^{\infty} \sigma(2 n-1) x^{8 n-4} .
\end{aligned}
$$

Hence,

$$
F^{\prime}(x)=x^{-1} F(x)\left\{1+24 \sum_{1}^{\infty} \sigma(O(2 n)) x^{8 n}+8 \sum_{1}^{\infty} \sigma(2 n-1) x^{8 n-4}\right\} .
$$


Eliminating $F^{\prime}(x)$ between (5) and (6), and cancelling $x^{-1} F(x)$ in the resulting identity we have

$$
\sum_{0}^{\infty} r_{4}(n) x^{4 n}=1+24 \sum_{1}^{\infty} \sigma(O(2 n)) x^{8 n}+8 \sum_{1}^{\infty} \sigma(2 n-1) x^{8 n-4} .
$$

Equating coefficients of like powers of $x$ in the foregoing identity we thus prove our theorem.

REMARKS. Identities (1) and (2) are easy special cases of the Gauss-Jacobi triple-product identity, and accordingly must now be regarded as being part of the folklore. Identity (3) is not as familiar as the other two, but, as shown in [2], it, too, is an elementary result. Finally, we should mention that identity (3) can also be deduced as an easy consequence of Jacobi's triangular-number identity.

\section{REFERENCES}

1. L. E. Dickson, History of the theory of numbers, Vol. 2, Chelsea, New York, 1952.

2. J. A. Ewell, Completion of a Gaussian derivation, Proc. Amer. Math. Soc. 84 (1982), 311-314.

3. G. H. Hardy and E. M. Wright, An introduction to the theory of numbers, 4th ed., Clarendon Press, Oxford, 1960.

Department of Mathematical Sciences, Northern Illinois University, DEKALB, ILLINOIS 60115 